\title{
A Polymethylvinylsiloxane/Low Density Polyethylene Blending Melt: Dynamic Rheological Behavior and Relaxation Time
}

\author{
Hua Yong Liao ${ }^{1,2 *}$, Ya Qian $\mathrm{Li}^{1,2}$, Chun Lin Liiu ${ }^{1,2}$ and Guo Liang Tao ${ }^{1,2}$ \\ ${ }^{1}$ Jiangsu Key Laboratory of Environmentally Friendly Polymeric Materials, School of Materials Science and Engineering, China \\ 2Jiangsu Collaborative Innovation Center of Photovolatic Science and Engineering, Changzhou University, China
}

Submission: January 20, 2020; Published: February 04, 2020

*Corresponding author: Hua Yong Liao, Jiangsu Key Laboratory of Environmentally Friendly Polymeric Materials, School of Materials Science \& Jiangsu Collaborative Innovation Center of Photovolatic Science and Engineering, Changzhou University, China

\begin{abstract}
The dynamic rheological behavior of a polymethylvinylsiloxane (PMVS)/ low density polyethylene (LDPE) blending melts at 150,180 and $210^{\circ} \mathrm{C}$ is investigated by small amplitude oscillatory shear on a rotational rheometer. LDPE has higher viscosity than PMVS at three temperatures, which affects the morphology and rheological response of the blends. The LDPE-rich blends have smaller dispersed droplets and longer relaxation time than the PMVS-rich blends. Higher temperature means less viscosity as well as dynamic modulus difference between the LDPE-rich blend and the PMVS-rich blend. Increasing temperature fastens relaxation of polymer samples thus relaxation time at higher temperature is shorter than that at low temperature. Phase separation can be shown at some weight ratio of PMVS/LDPE through the failure of time-temperature superposition principle and double peaks of relaxation time including the form relaxation time peak due to relaxation of dispersed droplets.

Keywords: Rheology; Blend; Relaxation Time; polymethylvinylsiloxane; Rheological Analysis; Viscosity; Elasticity

Abbreviations: PMVS: Polymethylvinylsiloxane; LDPE: Low Density Polyethylene; PP: Polypropylene; PA6: Polyamide 6; EPDM: EthylenePropylene-Diene; PCL: Polycaprolactone
\end{abstract}

\section{Introduction}

In recent years rheology of polymer blends attracts lots of researchers, and blending is an easy and convenient method to obtain new and valuable polymer materials. Most blends are immiscible, and the physical properties are affected by many factors such as weight ratios, viscosity ratio, interfacial tension [1,2] and shear strength etc. Rheological analysis is a powerful tool to relate the rheological behavior and phase structure at different environments. In dynamic shear on polymer blend, a balance between the breakup and coalescence affects the morphology and rheological response. Coalescence mainly depends on two parameters, one is the number of droplet collisions, another is the ability of the blend to drain the film of matrix phase, which is entrapped between two droplets of the dispersed phase until the critical thickness for coalescence to happen is reached [3]. Increase of concentration and droplet size of dispersed phase produces higher number of droplets collisions. Two factors affect film drainage: interfacial tension and viscosity of the matrix. Droplets with high viscosity are easy to remain spherical during flow, while droplets with low viscosity are likely to deform. Decrease of droplet coalescence will lead to smaller particle size in compatibilized blends [4]. Droplets will break when interfacial stress cannot counterbalance the shear stress anymore.

Viscosity ratio of blend's phases affects mainly the dynamic modulus at low frequency since geometrical relaxation of the droplets of dispersed phase brings about long-time relaxation. Elasticity affects phase behavior of immiscible polymer blends besides viscosity [5-7] and a corresponding model [8] has been developed to describe elastic contribution to the interfacial tension. Rheology and morphology of a polypropylene/polyamide 6 (PP/PA6) blends compatibilized with maleic anhydride grafted polypropylene (PP-g-MAH) was investigated by Shi et al. [9]. They found the morphology of the blend was of an emulsion-in-emulsion type, i.e., PA6 particles dispersed in the PP matrix themselves contained PP or PP-g-MAH inclusions. The emulsion-in-emulsion morphology became visible when PA6 content was high, for example, the ternary blend with PP/PP-g-MAH/PA6 was 35/35/30. In our recent work [10], a polypropylene(PP)/ high density polyethylene(HDPE) blend compatibilized with an Ethylene-Propylene-Diene Monomer block copolymer(EPDM) has similar morphology and rheology. This emulsion-in-emulsion structure is in 
fact the so-called "core-shell". A Core-shell morphology (core of HDPE and shell of EPDM) in PP matrix for a polypropylene (PP)/ ethylene-propylene-diene (EPDM) rubber/high density polyethylene (HDPE) 70/20/10 blend was successfully predicted by minimum free energy model [11]. Also, another core-shell morphology (core: HDPE, shell: EPDM-g-MA in PA6 matrix) was observed in PA6/EPDM-g-MA/HDPE ternary blends [12].

Polymethylvinylsiloxane (PMVS) $[13,14]$ is a material with outstanding properties and has been widely used in industries, such as thermal shielding, press pads, and weather resistance, etc. The performance and radiation crosslinking of polycaprolactone (PCL) and polymethylvinylsiloxane (PMVS) blends has been investigated by Zhu and co-workers [15]. But researches on rheological behavior on polymer blends about silicon rubber are not enough. In this paper, dynamic rheological behavior and relaxation time of a PMVS/LDPE binary blend were investigated. It was found viscosity difference between the two components and temperature affect greatly the morphology and rheological response, relaxation time of the blends. The results are interesting and useful in processing of silicon rubber materials.

\section{Experimental}

\section{Materials and Methods}

PMVS 110-2, manufactured by Jilin Jihui Industry and Commerce Co., Ltd (Jilin, China). Its mass average and number-average molar masses are $\mathrm{Mw}=570,000 \mathrm{~g} / \mathrm{mol}$ and $\mathrm{Mn}=370,000 \mathrm{~g} / \mathrm{mol}$, respectively. LDPE 2102 TN26 is produced by Qilu Petro. Co. Lt$\mathrm{d}$ (Shandong China). Its mass average and number-average molar masses are $\mathrm{Mw}=190,000 \mathrm{~g} / \mathrm{mol}$ and $\mathrm{Mn}=23,000 \mathrm{~g} / \mathrm{mol}$, respectively.

\section{Preparation of Blends}

Results and Discussion
The materials were dried at $80{ }^{\circ} \mathrm{C}$ for $12 \mathrm{~h}$ in an electric blast drying oven, then were mixed in an internal mixer with the weight ratio PMVS/LDPE of 10/90, 30/70, 50/50, 70/30 and 90/10 at $150{ }^{\circ} \mathrm{C}$ for 30 minutes. The rotating speed of the internal mixer was $30 \mathrm{r} / \mathrm{min}$. After that the samples were scraped off the internal mixer, then were cooled down at room temperature. The sample disks with diameter $25 \mathrm{~mm}$ and thickness $2 \mathrm{~mm}$ for rheological measurement were prepared by molding with a laboratory press at $150{ }^{\circ} \mathrm{C}$ and under $10 \mathrm{MPa}$ for $10 \mathrm{~min}$, and then cooled in the atmosphere.

\section{Measurement}

Microscopic images: Melt the blending sample at $210^{\circ} \mathrm{C}$ between cover glasses on an electric stove and made into a piece of film, then cooled the sample film to room temperature at nitrogen atmosphere. Put the prepared film onto the objective table of a polarization microscope and observed the microscopic photos at unpolarized mode at proper magnification and save the photos on a computer. The average dispersed phase size is calculated by a software Nano Measurer 1.2.0.

Rheological measurement: small amplitude oscillatory shear was applied by using a rotational rheometer MCR301 made by Anton Paar GmbH of Germany with two parallel plates. The diameter of the plate is $25 \mathrm{~mm}$, the gap of the two plates was $1 \mathrm{~mm}$. The temperature in the experiment was $150{ }^{\circ} \mathrm{C}, 180^{\circ} \mathrm{C}$ and $210^{\circ} \mathrm{C}$, and the angular frequency was from $0.028 \mathrm{rad} \cdot \mathrm{s}^{-1}$ to $300 \mathrm{rad} \cdot \mathrm{s}^{-1}$ in ascending order. The measurement was under nitrogen gas to avoid the oxidization of the samples. Before the start of the measurement the samples were kept for $5 \mathrm{~min}$ between the gap of the two plates to eliminate thermal history. The linear viscoelastic regime was determined by applying strain sweeping.

\section{Results and Discussion}

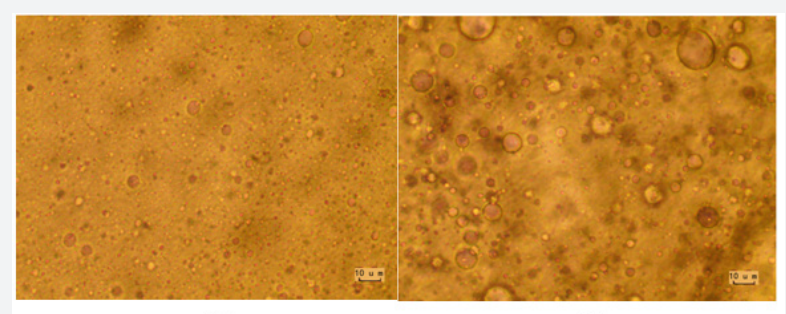

(a)

(b)

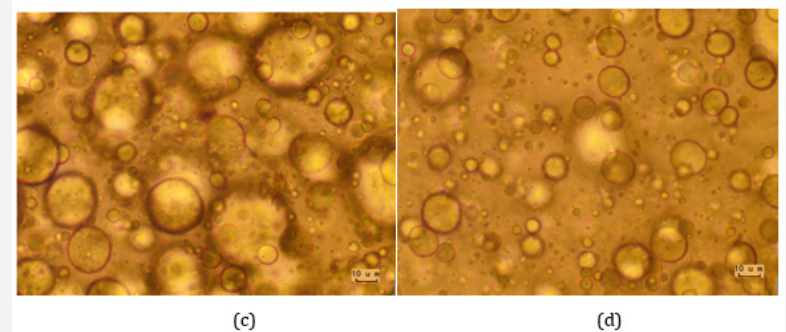

(c)

(d)

Figure 1: Microscopic images of the PMVS/LDPE blends (a)10/90, (b)30/70, (c)70/30 and (d)90/10, respectively. 
Figure 1 shows microscopic images of the PMVS/LDPE blends with weight ratio 10/90,30/70, 70/30 and 90/10, respectively. The average diameter of the dispersed droplets is $14.0 \mu \mathrm{m}, 19.54 \mu \mathrm{m}, 7.16 \mu \mathrm{m}$ and $3.43 \mu \mathrm{m}$, respectively. For the LDPErich blends, such as $10 / 90$ and $30 / 70$, the matrix is LDPE and the dispersed phase is PMVS. For the PMVS-rich blends, such as $70 / 30$ and $90 / 10$ blends, LDPE is the dispersed phase and PMVS is the matrix. The viscosity of LDPE is much higher than that of PMVS, as shown in Figure 2-4. Thus, it is much easier for PMVS to breakup in LDPE matrix, and more difficult for LDPE to breakup in PMVS matrix. In another word, the more viscous discrete LDPE droplets in the 70/30 PMVS/LDPE sample are more easily to be coarsened. The balance between the breakup and coalescence affects the morphology and rheological response [16].

Figure 2-4 show the dynamic rheological behavior of the PMVS/LDPE blending melts including the pure components PMVS and LDPE at 150,180 and $210^{\circ} \mathrm{C}$. At three temperatures LDPE has higher viscosity than PMVS, and the viscosity of the blend de- creases as the weight ratio of the PMVS/LDPE increases except the 50/50 blend. All the blends exhibit shear thinning behaviors in the range of applied angular frequencies. As the temperatures increases from 150 to $210{ }^{\circ} \mathrm{C}$, the viscosity of the blends drops, and the viscosity of the LDPE-rich blend drops much faster than the PMVS-rich blend, as shown in Figure 2-4. For example, the 10/90(PMVS/LDPE) blend has a viscosity of 21,000Pas,9040 Pas and 5190 Pas at 0.028rad/s, while the 90/10(PMVS/LDPE) blend has a viscosity of 6690Pas, 5010 Pas and 4780 Pas, respectively. This is understandable, since LDPE is a branched polymer, and PMVS is a liner polymer, the former is more sensitive to temperature than the latter. Higher temperature means less viscosity difference between the LDPE-rich blend and the PMVS-rich blend. The dynamic modulus of the blend has similar behavior with the viscosity. Due to the shape relaxation of discrete phase in the matrix, the dynamic modulus of the blends present much more difference at lower frequency, and the difference disappears gradually at high frequency [17].

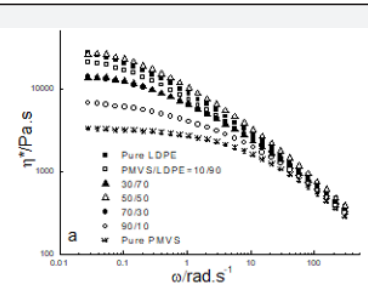

(a)

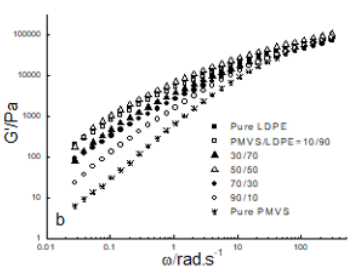

(b)

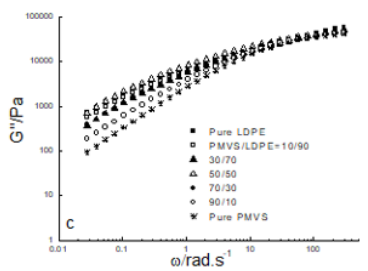

(c)

Figure 2: (a) Complex viscosity, (b) Storage modulus, and (c) Loss modulus vs angular frequency of PMVS/LDPE blends at $150{ }^{\circ} \mathrm{C}$.

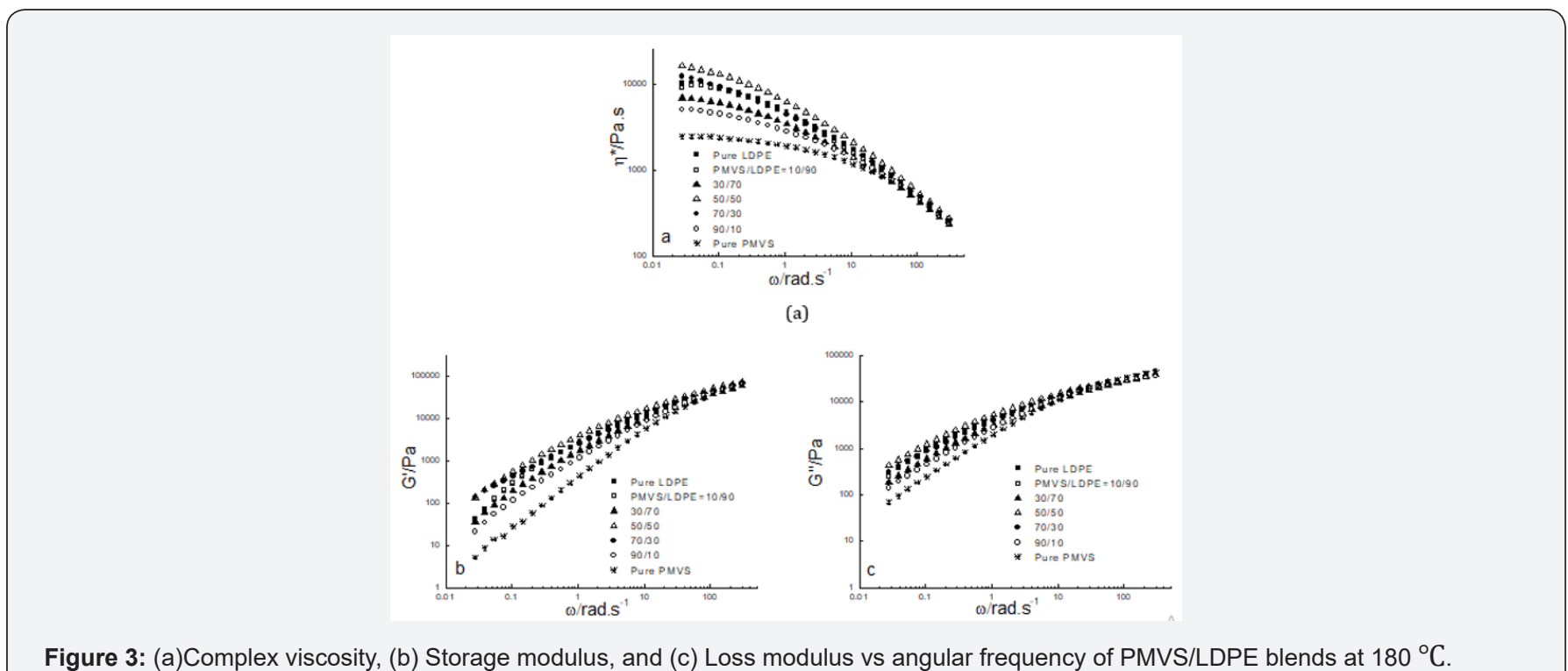

Figure 3: (a)Complex viscosity, (b) Storage modulus, and (c) Loss modulus vs angular frequency of PMVS/LDPE blends at $180{ }^{\circ} \mathrm{C}$. 


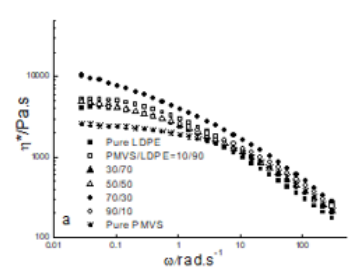

(a)

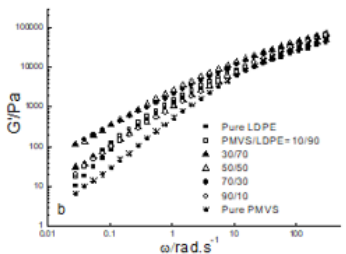

(b)

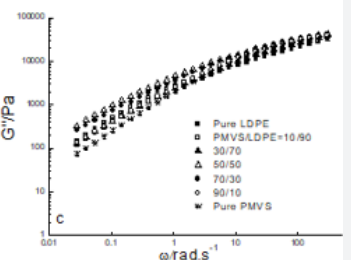

(c)

Figure 4: (a) Complex viscosity, (b) Storage modulus, and (c) Loss modulus vs angular frequency of PMVS/LDPE blends at $210^{\circ} \mathrm{C}$.

It is noticed the dynamic modulus of the 50/50 and 70/30 blend at low frequency is higher than that of pure PMVS and LDPE at $180^{\circ} \mathrm{C}$ and $210^{\circ} \mathrm{C}$. This means these two blends are immiscible $[18,19]$. The storage modulus of the two blends increases because the interfacial area of the blends is changed by the relaxation and deformation of dispersed phase droplets during dynamic rheological measurement. Comparing the storage modulus curve and loss modulus curve at three temperatures as shown in Figure 2-4, it can be seen the loss modulus is higher than the storage modulus at low frequency. The storage modulus is more sensitive to interfacial tension than loss modulus at low frequency. It is worth mentioning that the PMVS used in the blends is uncured sample and can flow easily at high temperatures. Vulcanization can increase the strength of the PMVS sample as well as the blends, which is not the interest of this work $[15,20]$.

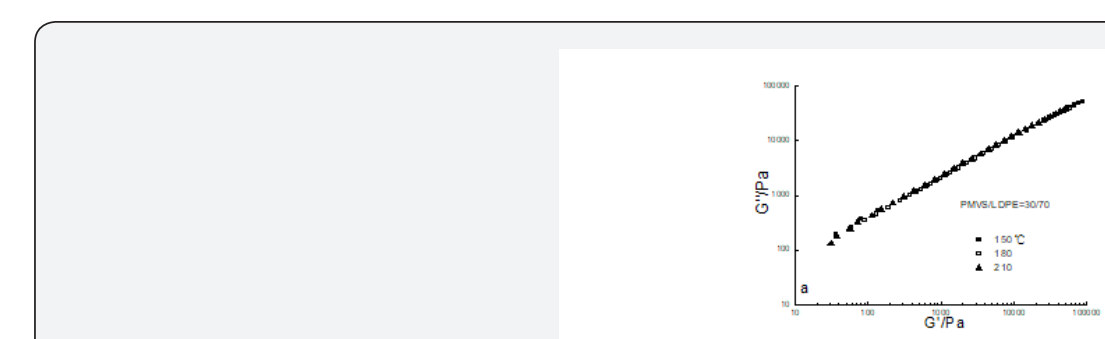

(a)

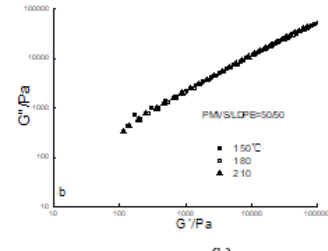

(b)

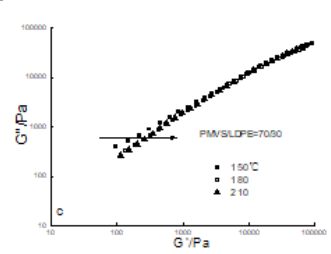

(c)

Figure 5: Cole-Cole diagram of G" vs. G' for PMVS/LDPE blends at 150,180 , and $210^{\circ} \mathrm{C}$.

The Cole-Cole diagram G" versus G' data at three temperatures for the 30/70,50/50 and 70/30 PMVS/LDPE blends were shown in Figure 5 respectively. It is obvious the G'-G' data at three temperatures overlapped well for the $30 / 70$ blend. But for the $50 / 50$ blend the data at low modulus somewhat dispersed. The dispersion phenomenon was more evident for the 70/30 blend. The invalidity of time-temperature superposition principle means phase separation of the blends [21]. As temperature increases, G' value increases with a fixed G" (see the arrow in Figure 5c), which has a similar trend to the work of $\mathrm{Wu}$ and co-workers [17]. The estimated interfacial tension of PMVS/LDPE blend at 150,180 and $210{ }^{\circ} \mathrm{C}$ is $6.07,6.53$ and $7.06 \mathrm{mN} / \mathrm{m}$ respectively $[22,23]$. Thus, the two polymers are immiscible. It is more difficult for more viscous LDPE droplets to disperse in less viscous PMVS matrix. The 10/90 blend and 90/10 blend have similar trends with the 30/70, and the 70/30 blend respectively, which are not shown in Figure 5. 


\section{Relaxation Time}

Table 1 lists the characteristic frequency and relaxation time of the blends when storage modulus( $\left.G^{\prime}\right)$ equals to loss modulus $\left(G^{\prime \prime}\right)$. The characteristic relaxation time is the reciprocal of the characteristic frequency. Firstly, comparing the relaxation time of pure component, it is clear that relaxation time of LDPE is longer than PMVS at the same temperature. It can be acceptable since LDPE has much higher viscosity than PMVS, as seen in Figure 2-4a. As the content of PMVS increases, relaxation time of the PMVS/LDPE blends drops gradually at a fixed temperature. In another word, the LDPE-rich blend has longer relaxation time than
PMVS-rich blend. As temperature increases, relaxation time of the sample decreases because polymer chains relax faster at higher temperature. The relaxation time listed in Table 1 is only some characteristic relaxation time. In fact, polymer has a relaxation time spectrum rather than one relaxation time. The weighted relaxation time spectrum of the blending melts can be obtained by using the approximation formula of dynamic modulus [24].

$$
\begin{gathered}
H(\tau)=G^{\prime}\left[d \log G^{\prime} / d \log \omega-\frac{1}{2}\left(d \log G^{\prime} / d \log \omega\right)^{2}\right. \\
\left.-(1 / 4.606) d^{2} \log G^{\prime}(d \log \omega)^{2}\right]_{1 / \omega=\tau / \sqrt{2}}
\end{gathered}
$$

Table 1: Relaxation time(s) of the blends when storage modulus(G')=loss modulus (G") .

\begin{tabular}{|c|c|c|c|c|c|c|c|}
\hline PMVS/LDPE & $\mathbf{0 / 1 0 0}$ & $\mathbf{1 0} / \mathbf{9 0}$ & $\mathbf{3 0 / 7 0}$ & $\mathbf{5 0 / 5 0}$ & $\mathbf{7 0 / 3 0}$ & $\mathbf{9 0 / 1 0}$ & $\mathbf{1 0 0 / 0}$ \\
\hline $150^{\circ} \mathrm{C}$ & 0.248 & 0.275 & 0.082 & 0.298 & 0.082 & 0.033 & 0.054 \\
\hline $180^{\circ} \mathrm{C}$ & 0.078 & 0.097 & 0.038 & 0.168 & 0.061 & 0.026 & 0.012 \\
\hline $210^{\circ} \mathrm{C}$ & 0.032 & 0.043 & 0.018 & 0.068 & 0.035 & 0.018 & 0.017 \\
\hline
\end{tabular}

Figure 6 shows the weighted relaxation time spectrum of the PMVS/LDPE blends. The shape of the spectrum curves at three temperatures are similar but with different intensities. Higher temperature corresponds to lower intensity. In order to analyze the relaxation time spectrum of the blends at different temperatures, (Table 2) lists the peak relaxation time. Basically, the LDPErich blend has longer relaxation time than PMVS-rich blend. This agrees with that shown in Table 1 . The 50/50 and 70/30 blends show two peaks of relaxation time at 180 and $210^{\circ} \mathrm{C}$. The relax- ation time of the pure PMVS is about 0.13-0.18s. The relaxation time of pure LDPE is about 2.56-18.68s at different temperatures, as shown in Table 2. Some relaxation time listed in Table 2 is more than 18.68s, it is neither that of PMVS nor of LDPE. This relaxation time is the secondary peak, which corresponds to the longest form relaxation time of dispersed phase droplets due to interfacial tension. Appearance of two relaxation time peaks also means phase separation of the blends, which is accordance with the failure of time-temperature superposition principle shown in Figure 5.

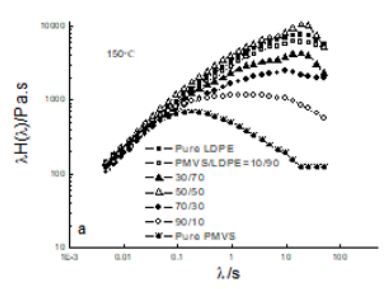

(a)

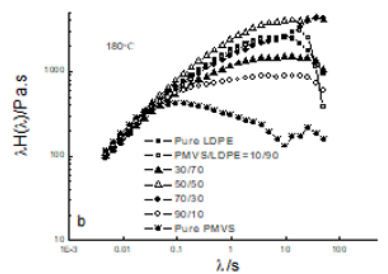

(b)

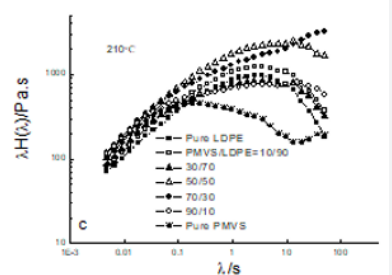

(c)

Figure 6: Weighted relaxation time spectra of the PMVS/LDPE blends at 150,180 and $210^{\circ} \mathrm{C}$.

Not all the blends show the longest form of relaxation time. In the work of Shi and co-workers [25] with various PP/PA6 uncompatibilized blends and PP/PP-g-MAH/PA6 compatibilized blends, both PP and PA6 show individual relaxation time peak, and some blends show a third peak at the longest time corresponded to the form relaxation time. In the present work, PMVS/LDPE blends did not show obviously individual peak relaxation time like that [26], possibly because the two peak relaxation time merged together, 
even the three peaks of relaxation time including the form relaxation time merged. Relaxation time can also see from the peak of dynamic viscosity ( $\eta$ " vs $\omega$ ) curve [27]. It was reported [28] in- terfacial force between PP and PS was affected by addition of the silica filler. Similar investigation about silica filler upon polymer blends can be found in the literature [29-30].

Table 2: Peak relaxation time of the blends (s).

\begin{tabular}{|c|c|c|c|c|c|c|c|}
\hline PMVS/LDPE & $\mathbf{0 / 1 0 0}$ & $\mathbf{1 0 / 9 0}$ & $\mathbf{3 0} / \mathbf{7 0}$ & $\mathbf{5 0 / 5 0}$ & $\mathbf{7 0 / 3 0}$ & $\mathbf{9 0 / 1 0}$ & $\mathbf{1 0 0 / 0}$ \\
\hline $150^{\circ} \mathrm{C}$ & 18.68 & 13.47 & 18.68 & 18.68 & 9.62 & 2.56 & 0.18 \\
\hline $180^{\circ} \mathrm{C}$ & 9.62 & 18.68 & 13.47 & $13.47 / 36.26$ & $4.96 / 36.26$ & 13.47 & 0.13 \\
\hline $210^{\circ} \mathrm{C}$ & 2.56 & 2.56 & 2.56 & 13.47 & $6.7 / 50.51$ & 13.47 & 0.18 \\
\hline
\end{tabular}

\section{Conclusion}

Dynamic rheological behavior of the PMVS/LDPE blends at 150,180 and $210^{\circ} \mathrm{C}$ is investigated through small amplitude oscillatory shear. The more viscous LDPE is more difficult to breakup in PMVS matrix, thus the LDPE-rich blends have smaller dispersed droplets than the PMVS-rich blends. Higher temperature decreases the viscosity difference between the LDPE-rich blend and the PMVS-rich blend. The 50/50 and 70/30 PMVS/LDPE blends exhibit invalidity of time-temperature superposition principle. Furthermore, the LDPE-rich blends have higher viscoelasticity and longer relaxation time than the PMVS-rich blends. The blends at higher temperature have shorter relaxation time and lower relaxation time spectrum. Some relaxation time spectrum of the PMVS/ LDPE blends show only one peak, the spectrums of the 50/50 and 70/30 PMVS/LDPE blends show a main peak and a longest form relaxation time. These cases infer the PMVS/LDPE blends are immiscible.

\section{References}

1. Samuel C, Parpaite T, Lacrampe MF (2019) Melt compatibility between polyolefins: Evaluation and reliability of interfacial/surface tensions obtained by various techniques. Polymer Testing 78: 105995.

2. Elias L, Fenouillot F, Majeste JC, Philippe C (2007) Morphology and rheology of immiscible polymer blends filledwith silica nanoparticles. Polymer 48: 6029-6040.

3. Lepers JC, Favis BD, Lacroix C (1999) The influence of Partial emulsification on coalescence suppression and interracial tension reduction in PP/PET blends. J Polym Sci, Part B:Polym Phys 37(9): 939-951.

4. Macaubas PHP, Demarquette NR (2001) Morphologies and interfacial tensions of immiscible polypropylene/polystyrene blends modified with triblock copolymers. Polymer 42(6): 2543-2554.

5. Steinmann S, Cronski W, Friedrich C (2001) Co-continuous polymer blends: influence of viscosity and elasticity ratios of the constituent polymers on phase inversion. Polymer 42: 6619-6629.

6. Steinmann S, Cronski W, Friedrich C (2002) Quantitative rheological evaluation of phase inversion intwo-phase polymer blends with cocontinuous morphology. Rheol Acta 41: 77-86.

7. Bourry D, Favis BD (1998) Cocontinuity and phase inversion in HDPE/ PS blends: influence of interfacial modification and elasticity. J Polym Sci B 36(11): 1889-1899.

8. Van Oene H (1972) Modes of dispersion of viscoelastic fluids in flow. J Colloid Interface Sci 40: 44867.
9. Shi DA, Ke Z, Yang JH (2002) Rheology and morphology of reactively compatibilized PP/PA6 blends. Macromolecules 35: 8005-8012.

10. Liao HY, Tao GL, Liu GL, Gong FH (2016) A polypropylene/high density polyethylene blend compatibilized with an ethylene-propylenediene monomer block copolymer: Fitting dynamic rhological data by emulsion models with a physical scheme. Journal of Applied Polymer Science 133(30): 43709.

11. Li LP, Yin B, Yang MB (2011) Morphology prediction and the effect of core-shell structure on the rheological behavior of PP/EPDM/HDPE ternary blends. Polym Eng Sci 51(12): 2425-2433.

12. Li LP, Yin B, Zhou Y, Lie, Yan G (2012) Characterization of PA6/EPDMg-MA/HDPE ternary blends: The role of core-shell structure. Polymer 53: 3043-3051.

13. Liu D, Song L, Song H, Lu A, Wang Y, et al. (2018) Correlation between mechanical properties and microscopic structures of an optimized silica fraction in silicone rubber. Composites Science and Technology 165: 373-379.

14. Liu D, Chen J, Song L (2017) Parameterization of silica-filled silicone rubber morphology: A contrast variation SANS and TEM study. Polymer 120: 155-163.

15. Zhu G, Xu S, Wang J, Zhang L (2006) Shape memory behaviour of radiation-crosslinked PCL/PMVS blends. Radiation Physics and Chemistry 75: 443-448.

16. Lee HM, Park OO (1994) Rheology and dynamics of immiscible polymer blends. J Rheol 38: 1405-1425.

17. Wu DF, Zhang YS, Zhang M, Zhou W (2008) Phase behavior and its viscoelastic response of polylactide/poly(e-caprolactone) blend. Eur Polym J 44(7): 2171-2183.

18. Gramespacher H, Meissner J (1992) Interfacial-tension between polymer melts measured by shear oscillations of their blends. J Rheol 36: 1127-1141.

19. Graebling D, Muller R, Palierne JF (1993) Linear viscoelastic behavior of some incompatible polymer blends in the melt. Interpretation of data with a model of emulsion of viscoelastic liquids. Macromolecules 26: 320-329.

20. Harkous A, Colomines G, Leroy E, Pierre M (2016) The kinetic behavior of Liquid Silicone Rubber: A comparison between thermal and rheological approaches based on gel point determination. Reactive and Functional Polymers 101: 20-27.

21. Li RM, Yu W, Zhou CX (2006) Phase behavior and its viscoelastic response of poly(methyl methacrylate) and poly(styrene-co-maleic anhydride) blend systems. Polym Bull 56: 455-466

22. Contino M, Andena L, Rink M (2018) Time-temperature equivalence in environmental stress cracking of high-density polyethylene. Engineering Fracture Mechanics 203: 32-43. 
23. Pini T, Briatico Vangosa F, Frassine R, Rink M (2017) Fracture toughness of acrylic resins: viscoelastic effects and deformation mechanisms. Polym Eng Sci 58(3): 369-76.

24. Wu RJ (1998) Polymer interface and adhesion. Science Press, Beijing, China.

25. Zhang ZY, Qiao JL (1993) Method of estimating interfacial tension in polymer systems. Acta Polymerica Sinca 3: 343-347.

26. Tschoegl NW (1989) The phenomenological theory of linear viscoelastic behavior. Springer, Berlin, Germany.

27. Shi D, Hu G , Ke Z, Li K (2006) Relaxation behavior of polymer blends with complex morphologies: Palierne emulsion model for uncompatibilized and compatibilized PP/PA6 blends. Polymer 47(13) 4659-4666.
28. Elias L, Fenouillot F, Majeste JC (2007) Morphology and rheology of immiscible polymer blends filled with silica nanoparticles. Polymer 48: 6029-6040.

29. Kong M, Huang Y, Chen G, Yang Q, Li G (2011) Retarded relaxation and breakup of deformed PA6 droplets filled with nanosilica in PS matrix during annealing. Polymer 52(22): 5231-5236.

30. Wang J, Tang Q, Ren X (2019) Selectively localized nanosilica particles at the phase interface of PS/PA6/nanosilica composites with cocontinuous structure via reactive extrusion. Composites Science and Technology 172: 125-133.

Your next submission with Juniper Publishers will reach you the below assets

- Quality Editorial service

- Swift Peer Review

- Reprints availability

- E-prints Service

- Manuscript Podcast for convenient understanding

- Global attainment for your research

- Manuscript accessibility in different formats ( Pdf, E-pub, Full Text, Audio)

- Unceasing customer service

Track the below URL for one-step submission https://juniperpublishers.com/online-submission.php 